\title{
Characterization of PAN/ATO nanocomposites prepared by solution blending
}

\author{
WEI PAN* and HANTAO ZOU ${ }^{\dagger}$ \\ Institute of Materials and Chemical Engineering, Zhongyuan University of Technology, Zhengzhou 450007, P. R. China \\ ${ }^{\dagger}$ Institute of Textile and Material, WuHan University of Science and Engineering, Wuhan 430073, P. R. China
}

MS received 9 January 2008; revised 19 May 2008

\begin{abstract}
Conducting nanocomposites of polyacrylonitrile (PAN) and antimony-doped tin oxide (ATO) were prepared by solution blending. Electrical properties of the nanocomposites were characterized by means of electrical conductivity measurements and the phase structures were investigated via scanning electron microscopy (SEM), X-ray diffraction (XRD), thermogravimetric analyses (TGA) and dynamical mechanical analysis (DMA). This study unveiled a remarkable, interpenetrating network of chainlike nano-ATO within the PAN matrix, consistent with the existence of connected conducting paths at content as low as $4 \mathrm{wt} \%$ of the nanoATO. The storage modulus of the nanocomposites increased with increasing content of ATO, due to formation of immobilized layer between polymer and filler. The interactions between ATO and PAN molecules resulted in high $\tan \delta$ for the PAN/ATO nanocomposites. Thermal stability of the nanocomposites was found remarkably enhanced by the incorporation of nano-ATO.
\end{abstract}

Keywords. Antimony doped tin oxide; polyacrylonitrile; nanocomposites; structure and properties.

\section{Introduction}

Polymers can be compounded with a variety of common and special fillers, reinforcements, and modifiers to yield specific properties in a wide range of applications. Among these additives are electrically and thermally conductive modifiers that can provide protection against static accumulation, electrostatic discharge and molding cycle reduction. Various fillers, some of them metallic, are used to produce the modification of conductive properties of the neat polymer. However, high concentrations of filler (e.g. $30 \%$ carbon black), are needed that can take a toll on physical and esthetical properties of the polymer: selection of conductive material has often involved compromises (Alexandre et al 2002; Causin et al 2006). The preparation of polymer composites by dispersion of small loadings of nanosized fillers in a polymeric matrix has lately attracted much attention in the academia and the industry for its potential in improving the performance of macromolecular materials. Nanoparticles may confer to the matrix much improved physical and mechanical properties with respect to traditional micron-sized fillers, due mainly to maximization of the polymer-filler interfacial region (Vaia and Wagner 2004).

Particularly interesting conductive filler is antimonydoped tin oxide (ATO) as it combines a good electrical conductivity with optical transparency (Kim et al 1994;

*Author for correspondence (panwei@zzti.edu.cn)
Ginley and Bright 2000; Lewis and Paine 2000; Rajpure and Kusumade 2000). ATO has been the focus of intensive studies due to its high-temperature, chemical and mechanical stabilities. At low Sb doping level, the conductivity of ATO is greatly increased as compared to pure tin oxide and can be varied easily by changing the $\mathrm{Sb}$ doping level (Shanthi et al 1980). When used as antistatic agent, ATO shows better performance than the currently used carbon blacks, metallic pigment and organic polymer binders (Orel et al 1992). ATO has been used to increase the electrical conductivity of polyvinyl acetateacrylate copolymer coatings (Sun and Francis 2003), gelatine and acrylate films (Wang and Anderson 1999), and urethane and polyester coatings (Palacio et al 2001). For the different matrices, the minimum amount of ATO needed to obtain the aimed increase in conductivity varied roughly from 5-20 vol\%.

Polyacrylonitrile (PAN) is one of the most important fibre-forming polymers and has been widely used because of its high strength, abrasion resistance, and good insect resistance (Pan et al 2005). However, the conductivity of PAN is $<10^{-14} \mathrm{~S} / \mathrm{cm}$ and the static problem restricts its further applications. PAN is usually synthesized using free radical polymerization. Usually they are copolymers of acrylonitrile and methyl acrylate, or acrylonitrile and methyl methacrylate. PAN has a melting point of about $319^{\circ} \mathrm{C}$, and it also decomposes at this temperature. So PAN is not suitable for melt process. Therefore, in our study, PAN/ATO nanocomposites were prepared by a solution-mixing method. The relationship between 
the electrical properties and the morphology of the composites was studied. The effects of mixing fillers on dynamic mechanical and thermal properties of the nanocomposites were also investigated.

\section{Experimental}

\subsection{Materials}

The PAN $\left(\mathrm{Mw}=5.5 \times 10^{4}\right)$ employed in this study was purchased from Shanghai Petroleum Chemical Co., Ltd (Shanghai, China). The untreated nano-ATO particles with specific surface area, $80 \mathrm{~m}^{2} / \mathrm{g}$, were purchased from Shanghai Huzheng Technology Co., Ltd. (Shanghai, China). The silane coupling agent (KH570, commercial grades) was supplied by Shanghai Yaohua Chemical Plant (Shanghai, China), which was used to treat the nano-ATO particles. Other agents and process assistants (commercial grades) were obtained from the market.

\subsection{Nano-ATO particle surface treatment}

Silane coupling agent $(0 \cdot 2 \mathrm{~g})$ was mixed with $5 \mathrm{~g}$ distilled water and $95 \mathrm{~g}$ absolute ethanol, and then $10 \mathrm{~g}$ ATO powder was added into the solvent mixture. The suspension was dispersed in an ultrasonicator for $1 \mathrm{~h}$. The suspension was refluxed at $120^{\circ} \mathrm{C}$ for $24 \mathrm{~h}$. After surface treatment, the nano-ATO particles were rinsed with ethanol three times in order to remove any unreacted silane coupling agent.

\subsection{Nanocomposites preparation}

The nano-ATO was dispersed in dimethyl sulfoxide (DMSO) to prepare a suspending dispersion with certain nano-ATO content. The PAN was dissolved in DMSO by heating to a backflow temperature of DMSO (about $80^{\circ} \mathrm{C}$ ). The ATO suspending dispersion was added drop by drop into the PAN solution at the backflow state, keeping the total concentration of the nano-ATO and the PAN as $18 \mathrm{wt} \%$ mass ratio. The mixture solutions were cast and left to dry in vacuum at $60^{\circ} \mathrm{C}$ for $48 \mathrm{~h}$.

\subsection{Testing methods}

2.4a Dynamic mechanical analysis: The dynamic storage modulus and the tangent of the ratio of the complex to real modulus $(\tan \delta)$ of sample were obtained from a TA $2980 \mathrm{DMA}$ at $3^{\circ} \mathrm{C} / \mathrm{min}$. The sample was $12 \times 2 \mathrm{~mm}^{2}$. The experiment was performed in a tension mode at a frequency of $1 \mathrm{~Hz}$.

2.4b Scanning electron microscopy: Morphological studies were performed by using SEM. Microphotographs were taken of the surface made by fracturing the speci- men in liquid nitrogen and then sputtered with gold. A JSM-5600LV scanning electron microscope was used for morphological observation.

2.4c X-ray diffraction: Wide angle X-ray diffraction was carried out using a BRUKER-AXC08 X-ray diffractometer and filtered $\mathrm{CuK} \alpha$ radiation. The diffraction patterns of the composite powder of the ATO and PAN were obtained by scanning the samples in an interval of $2 \theta=$ 5-60 degrees.

2.4d Thermal stability analysis: Thermogravimetric analyses of pure PAN and PAN/ATO nanocomposites were performed with a TA Instruments Du Pont 1090 at $10^{\circ} \mathrm{C} / \mathrm{min}$ under air atmosphere.

2.4e Electrical conductivity: Electrical conductivity of the blends was measured by the usual four-probe method, doing five measurements in three different samples of each composition, the standard deviation being always $<1 \%$.

\section{Results and discussion}

\subsection{Electrical conductivity}

The variation of the electrical conductivity of composites vs the content of nano-ATO is shown in figure 1. PAN is electrically nonconductive and has a conductivity of about $10^{-14} \mathrm{~S} / \mathrm{cm}$ in dry state at room temperature. It can be seen that the electrical conductivity of the PAN/ATO nanocomposite increases with increasing nanocomposites content. The incorporation of very small amount of nanoATO into the PAN matrix had dramatically increased the conductivity of composites with a sharp transition from an electrical insulator to an electrical semiconductor.

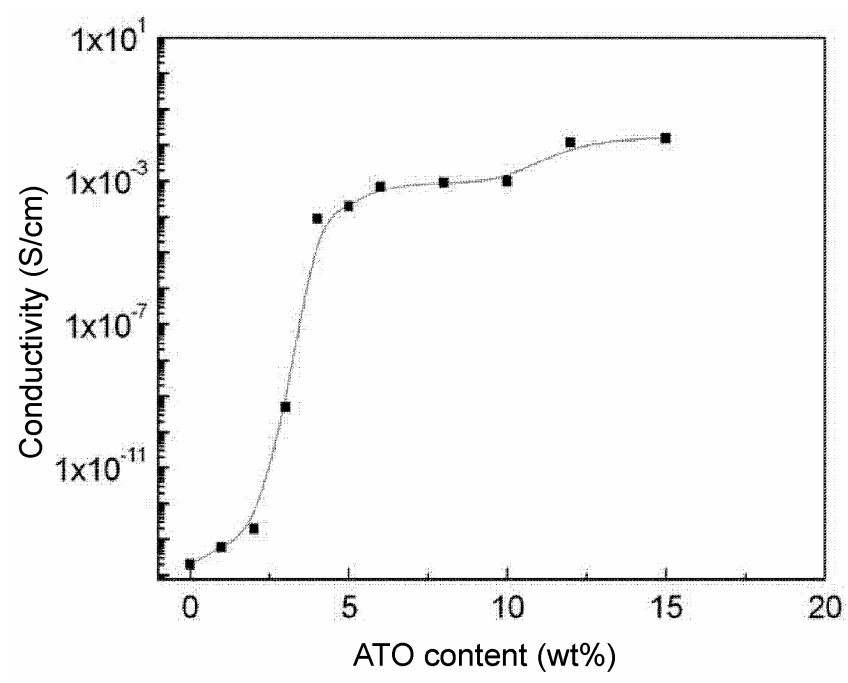

Figure 1. Electrical conductivity of PAN/ATO as a function of ATO. 

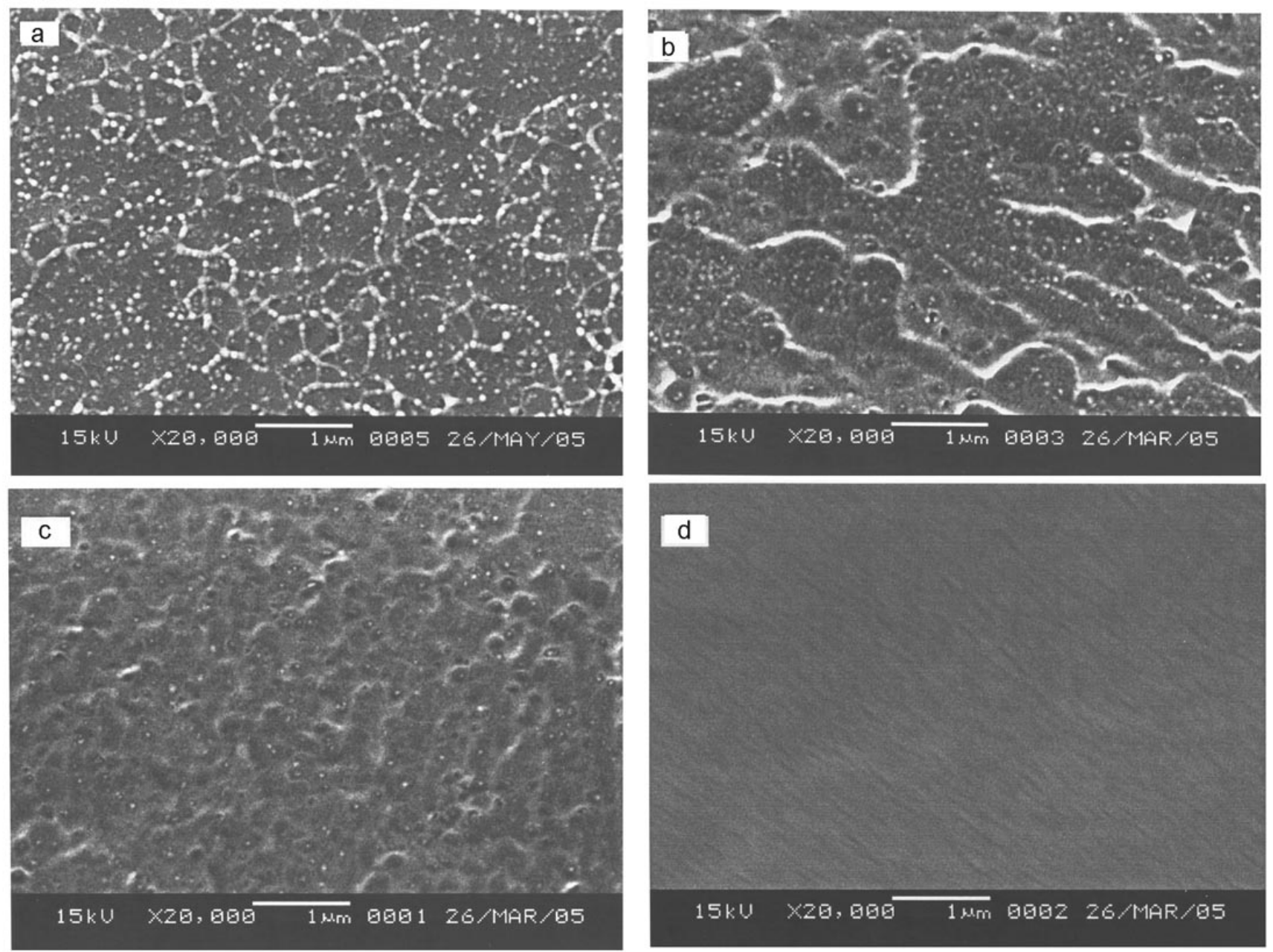

Figure 2. SEM micrographs of freeze fractured surfaces of PAN/ATO nanocomposites with different ATO contents: (a) 8 wt\%, (b) $4 \mathrm{wt} \%$, (c) $2 \mathrm{wt} \%$ and (d) $0 \mathrm{wt} \%$.

After the nano-ATO content reached $4 \mathrm{wt} \%$, the electrical conductivity of the nanocomposites tended to level off with further increase in nano-ATO content. This demonstrates the very low percolation threshold value for a conducting nanocomposite prepared with nano-ATO, which is much smaller than that of conventional conducting macro composites (Knite et al 2002).

The augmentation of the electrical conductivity can be ascribed to the nanoscale dispersion of nano-ATO within the polymer matrix and the formation of conducting networks at low filler loading. With the very low inorganic filler content, the resulting nanocomposites can remain the superior inherent mechanical strength of polymeric matrix. The nanocomposites exhibit as both high performance polymeric material and electrically conductive material.

\subsection{Morphology observation}

It was well established that the conductivity and percolation threshold of particle-filled polymers depend greatly on the interaction of polymer and conductive filler and the filler distribution. The morphology of PAN/ATO nanocomposite was studied to elucidate the structure responsible for the electrical conductivity behaviour. The cross-section SEM micrographs of the PAN/ATO nanocomposites containing $8,4,2$, and $0 \mathrm{wt} \%$ ATO, respectively are listed in figure 2 . The dark regions are related to the PAN phase while the bright regions to conductive nano-ATO phase.

Figure 2a shows the morphology of PAN/ATO nanocomposites containing $8 \mathrm{wt} \%$ ATO and this micrograph reveals a complex interconnected network of nano-ATO. Obviously, this nano-ATO morphology provides plenty of connected pathways for electron transport in the nanocomposite. As the concentration of nano-ATO in nanocomposite is decreased to $4 \mathrm{wt} \%$ (figure $2 \mathrm{~b}$ ), the interconnected network becomes less dense, although the nano-ATO remains aggregated into chain-like morphology indicative of connected pathways. Below $4 \mathrm{wt} \%$ ATO, the nano-ATO particles appear to be isolated and disconnected (see figure $2 \mathrm{c}$ ) corresponding to $2 \mathrm{wt} \%$ ATO. 


\subsection{Dynamic mechanical analysis}

Dynamic mechanical measurements are believed to be a good method for gaining insight into the effects exerted by the filler on the polymer matrix. These effects are displayed as a variation in dynamic storage modulus, a modification of the peak position and width of the relaxation spectrum as a consequence of filler-polymer interaction. Each peak marks a transition which is interpreted as released of a restricted movement either in the polymer chain or in lateral groups.

The storage modulus and $\tan \delta$ for the pure PAN and composite samples as a function of temperature are shown in figures 3 and 4 , respectively. In figure 3 we see the

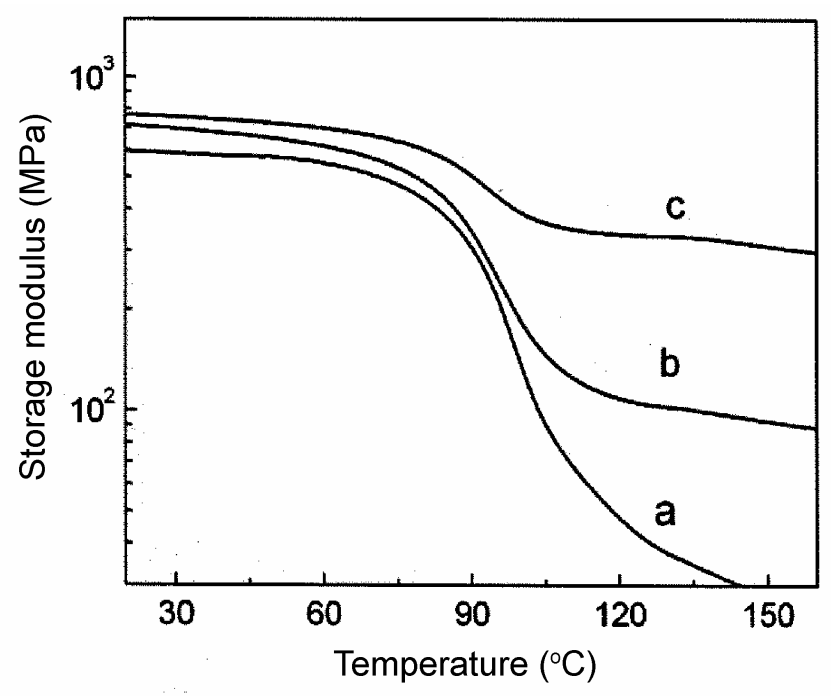

Figure 3. Storage modulus of PAN/ATO system with different ATO contents: a. $0 \mathrm{wt} \%$, b. $4 \mathrm{wt} \%$ and c. $8 \mathrm{wt} \%$.

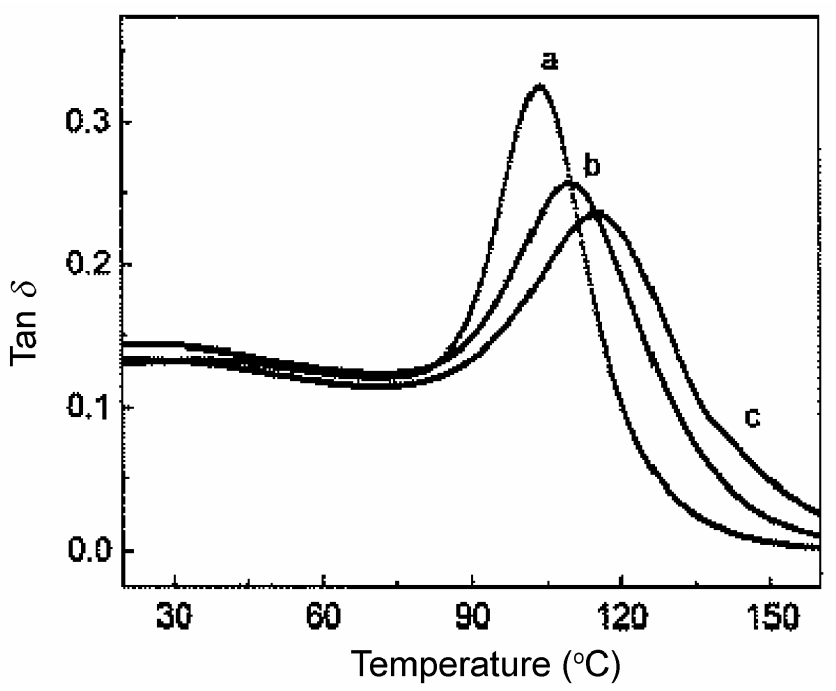

Figure 4. Tan $\delta$ of PAN/ATO system with different ATO contents: a. 0 wt $\%$, b. 4 wt $\%$ and c. 8 wt $\%$. values of storage modulus for filled samples consistently decrease at different rates as the temperature increases. The incorporation of the conducting fillers causes an increase of the dynamic storage modulus in all the intervals of temperatures studied. The system with greater total filler percentage ( $8 \mathrm{wt} \%$ ) shows the highest storage modulus with respect to pure polymer.

Figure 4 describes the relation between the glass transition temperatures ( $\tan \delta$ peak position) as a function of the nano-ATO content. It shows that $T_{\mathrm{g}}$ of the nanocomposites are higher than that of pure PAN. The magnitude of $\tan \delta$ peak decreased significantly, and the $\tan \delta$ peak broadened towards higher temperature with increasing nano-ATO. The motion of PAN molecules closer to the nano-ATO would be more constrained than those farther away from it. The broadening of the $\tan \delta$ peak to higher temperatures is attributed to this variation in interaction between PAN and nano-ATO. Similar results were reported by Tsagaropoulos and Eisenberg (1995) for the PVAc/silica, $\mathrm{PS} /$ silica, PMMA/silica, PDMS/silica and SBR/silica.

\subsection{Thermal analysis}

The TGA curves for the pure PAN and PAN/ATO nanocomposites with $8 \mathrm{wt} \%$ nano-ATO content in atmosphere are shown in figure 5 . There are two obvious weight loss peaks at $250-280^{\circ} \mathrm{C}$ and $570-600^{\circ} \mathrm{C}$ in the TGA curves. The first weight loss is caused by decarboxylation of the methylacrylate in Co-PAN and the second weight loss is due to thermo-oxidative degradation of PAN macromolecular chains. It is observed in figure 5 that the first thermal decomposition temperature of PAN/ATO with $8 \mathrm{wt} \%$ ATO content is around $274^{\circ} \mathrm{C}$, whereas that of pure PAN is only $252^{\circ} \mathrm{C}$. This clearly demonstrates that the PAN/ATO system is thermally more stable than the corresponding neat PAN system. This means that the incorporation of nano-ATO into PAN offers a stabilizing effect against the decomposition.

\subsection{Wide-angle X-ray diffraction study}

It is known that the crystal structure of the matrix has a key role in determining the properties of polymer-based composites (Tchmutin et al 2003; Radhakrishnan et al 2004). The XRD characterizations of nano-ATO (curve a), the pure PAN (curve d) and the PAN/ATO nanocomposites with 4 and $8 \mathrm{wt} \%$ of nano-ATO contents (curves b and c) were conducted (see figure 6). The X-ray patterns of the nano-ATO displayed the presence of five peaks at $2 \theta=26.5^{\circ}, 33.7^{\circ}, 37.7^{\circ}, 51.7^{\circ}$ and $54.7^{\circ}$ corresponding to the reflections of (110), (101), (200), (220) and (211) (Lili et al 2006). PAN shows only a scattering peak at $2 \theta=17^{\circ}$.

The XRD patterns of the PAN/ATO nanocomposites appear as both the characteristic peaks of the pure PAN 

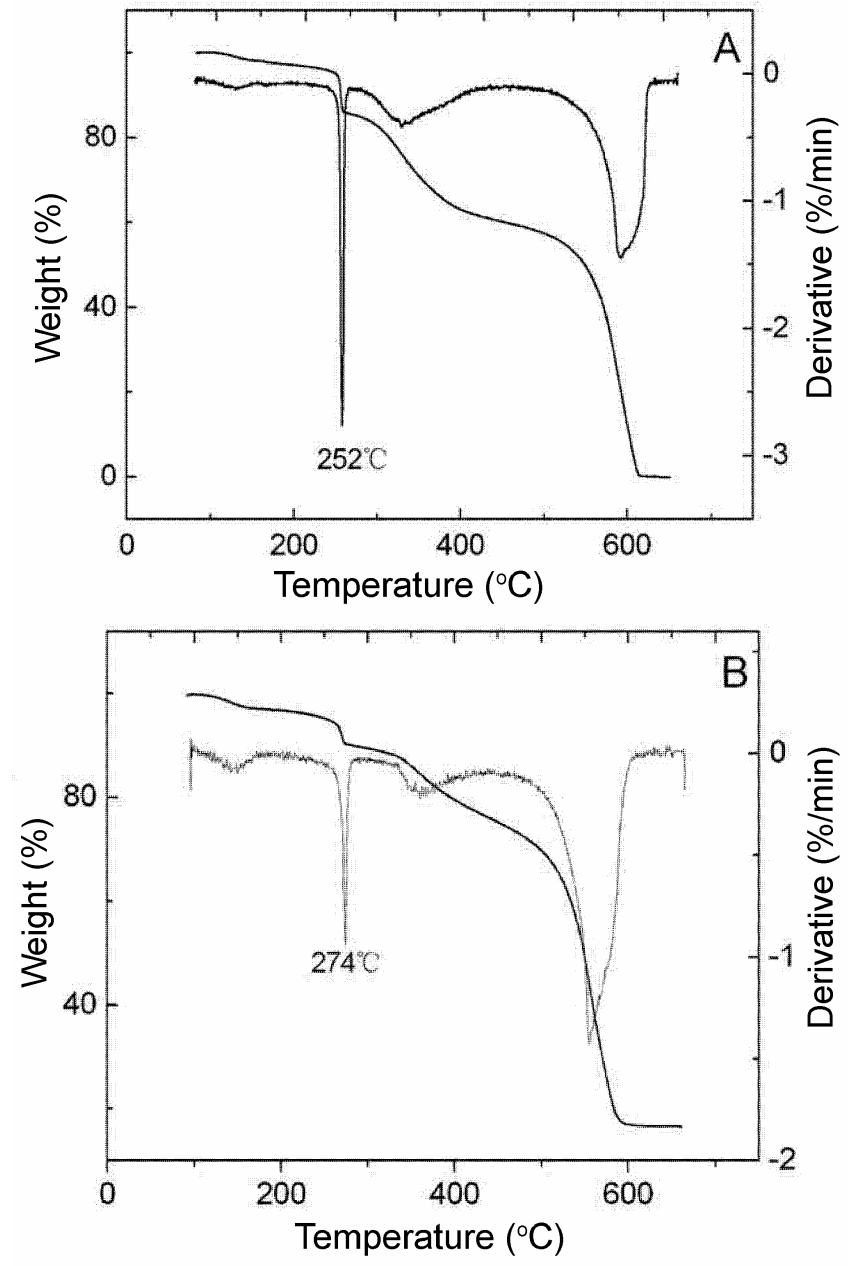

Figure 5. TGA curves of A. pure PAN and B. PAN/ATO $(8 \mathrm{wt} \%)$.

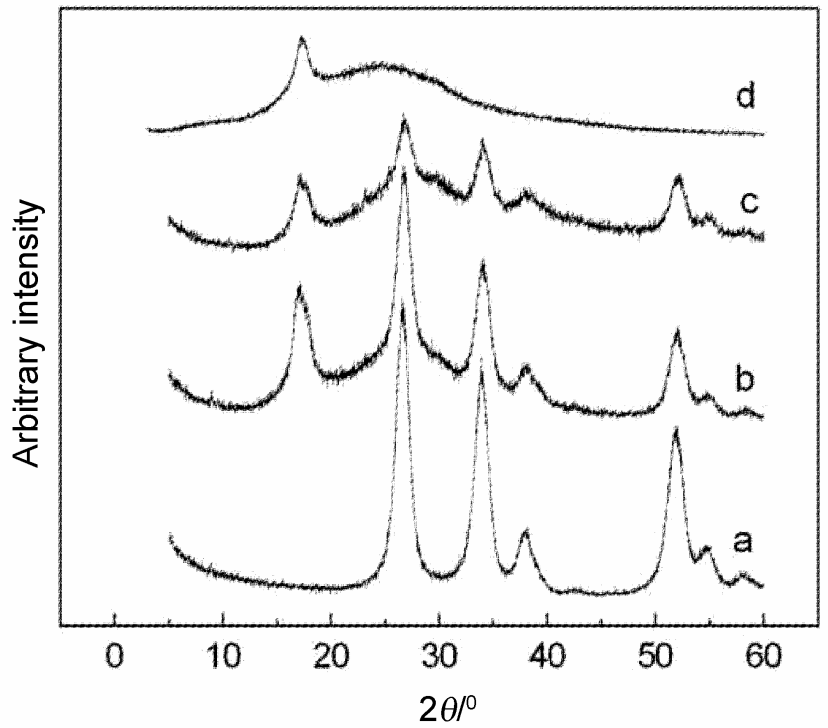

Figure 6. WXRD patterns of a. ATO, b. 4\% ATO content, c. 8 wt $\%$ ATO content and d. PAN. and nano-ATO. Moreover, the intensity of the peaks assigned to the nano-ATO in the composite increased with the increase of the content of nano-ATO. However, the position of the peaks corresponding to the two constituents of the nanocomposite was same as the individual PAN and nano-ATO, which illustrated that either the orientation of PAN chains or the structure of nano-ATO did not influence each other during the process of preparation.

\section{Conclusions}

Conductive PAN/ATO nanocomposites exhibiting a reduced low threshold have been successfully prepared by solution blending. Electrical properties of the composites were characterized and the morphology was investigated via SEM, XRD, TGA and DMA. In the PAN/ATO nanocomposites, the percolation threshold was about $4 \mathrm{wt} \%$. The nano-ATO delayed the decomposition of PAN. The storage modulus and the glass transition temperature of the polymer significantly increased with increasing nanoATO loading fraction.

\section{References}

Alexandre M and Dubois P 2000 Mater. Sci. Eng. 281

Causin Valerio, Marega Carla and Marigo Antonio 2006 Eur. Polym. J. 423153

Ginley D S and Bright C 2000 MRS Bull. 2515

Kim K H, Lee S W and Shin D W 1994 J. Am. Ceram. Soc. 77 915

Knite Maris, Teteris Valdis and Polyakov Boris 2002 Mater. Sci. Eng. C19 15

Lewis B G and Paine D C 2000 MRS Bull. 2525

Lili Li, Liming Mao and Xuechen Duan 2006 Mater. Res. Bull. 41541

Orel Z C, Orel B and Hodoscek M 1992 J. Mater. Sci. 27 313

Palacio M L, Wang Y and Gerberich W W 2001 J. Mater. Res. 163628

Pan Wei, Yang Sheng Lin and Li Guang 2005 Eur. Polym. J. 41 2127

Radhakrishnan S, Sonawane P and Pawashar N 2004 J. Appl. Polym. Sci. 93615

Rajpure K Y and Kusumade M N 2000 Mater. Chem. Phys. 64 184

Shanthi E, Dutta V, Banerjee A and Chopra K L 1980 J. Appl. Phys. 516243

Sun J and Francis L F 2003 J. Polym. Sci. Part B. Polym. Phys. 41744

Tchmutin I A, Ponomarenko A T, Krinichnaya E P and Kozub G I 2003 Carbon 411391

Tsagaropoulos G and Eisenberg A 1995 Macromol. 286066

Vaia R A and Wagner H D 2004 Mater. Today 732

Wang Y C and Anderson C 1999 Macromol. 326172 